\title{
Reference pricing in the presence of pseudo-generics*
}

\author{
Ricardo Gonçalves ${ }^{\dagger}$ \\ Faculdade de Economia e Gestão, Universidade Católica Portuguesa (Porto) \\ Vasco Rodrigues \\ Faculdade de Economia e Gestão, Universidade Católica Portuguesa (Porto) \\ Hélder Vasconcelos \\ Faculdade de Economia e Gestão, Universidade Católica Portuguesa (Porto)
}

March 2011

\section{PRELIMINARY DRAFT}

\begin{abstract}
We build on the analysis of Rodrigues et al. (2011) and look at the market impact of pseudogenerics under two possible reimbursement mechanisms: reference pricing and fixed percentage reimbursement. Reference pricing emerges as a preferable reimbursement mechanism, both from a consumer's perspective as well as from a welfare perspective, particularly when a pseudogeneric is present.
\end{abstract}

JEL Classification: I18, L13

Keywords: pseudo-generics, reference pricing, reimbursement mechanisms

*Financial support from Fundação para a Ciência e Tecnologia (project PTDC/EGE-ECO/100296/2008) is gratefully acknowledged.

${ }^{\dagger}$ Postal address: Faculdade de Economia e Gestão, Universidade Católica Portuguesa (Porto), Rua Diogo Botelho, 1327, 4169-005 Porto, Portugal. E-mail: rgoncalves@porto.ucp.pt. 


\section{Introduction}

As the patents of branded pharmaceuticals expire, generic competition becomes possible. The producers of the branded pharmaceutical can also market a generic version of their branded product, commonly known as pseudo-generics. Because such a strategy appears, at first sight, to be unprofitable - generics typically have a much lower price than the branded pharmaceutical and, thus, selling pseudo-generics could significantly reduce profit margins - Rodrigues, Gonçalves and Vasconcelos (2011) analyse the potential for anticompetitive effects associated with the presence of pseudo-generics in pharmaceutical markets. Their model assumes price-setting firms in a market with vertical (between branded and generic pharmaceuticals) and horizontal differentiation (between generics). They conclude that the presence of pseudo-generics raises the prices of all pharmaceuticals as they become a tool for the branded producer to soften competition between itself and generic producers.

This paper builds on Rodrigues et al. (2011) by considering a setting where a reimbursement mechanism is in place, i.e. where the consumer does not bear the full price of pharmaceutical products. We incorporate into the model two widely adopted reimbursement mechanisms: fixed percentage reimbursement (FPR) mechanism and a reference pricing (RP) mechanism. ${ }^{1}$ Under a FPR mechanism, the consumer supports a fixed percentage (copayment rate) of the pharmaceutical's price, whilst the government (or other third-party payers) is responsible for financing the remainder. By contrast, under a RP mechanism, within each cluster of pharmaceuticals, a 'reference' pharmaceutical is defined - typically a generic - and its price is defined as the 'reference price'.2 The government's contribution towards the purchase of pharmaceuticals is calculated on the basis of that reference price, i.e. if a consumer decides to purchase a more expensive pharmaceutical, the diference between that pharmaceutical's price and the reference price is fully borne by him. Naturally, reference pricing is only different from FPR when generic competition becomes possible, otherwise only one branded pharmaceutical exists which would automatically be the reference pharmaceutical.

There is a large consensus in the literature regarding the merit of reference pricing. For instance, Brekke et al. (2007) show that reference pricing leads to lower prices than FPR mechanisms. They further show that although from a social welfare perspective reference pricing is equivalent to FPR mechanisms, once a narrower welfare indicator - from a public payer's perspective - is adopted, which excludes firms' profits, the former is clearly superior. Merino-Castelló (2003) also shows that reference pricing enhances price competition and leads to significant reductions in the price of branded pharmaceuticals. Whilst also acknowledging the potential benefits of reference pricing, Miraldo (2009) notes that if firms anticipate the introduction of a reference pricing mechanism,

\footnotetext{
${ }^{1}$ For a detailed review of reference pricing, see López-Casasnovas and Puig-Junoy (2000).

${ }^{2}$ Several criteria could be used to cluster pharmaceuticals: chemical, pharmacological or therapeutic (Miraldo, 2009).
} 
they will optimally revise their prices upwards ahead of that introduction and thus significantly limit its expected effectiveness. Nevertheless, Brekke et al. (2009) present empirical evidence of significant price reductions for all pharmaceuticals following the introduction of reference pricing in Norway.

In line with Brekke et al. (2007), we find that reference pricing, with or without the presence of pseudo-generics, leads to lower prices and higher consumer surplus than FPR mechanisms. It is particularly interesting to note that, under a FPR mechanism, pharmaceutical firms effectively receive a public subsidy but consumers do not benefit from lower (effective) prices. By contrast, under reference pricing, it is consumers who receive a public subsidy, which allows them to increase their consumer surplus.

Moreover, adopting Brekke et al. (2007) narrower welfare measure - from a public payer's perspective - it is shown that RP fares better than FPR. In contrast to Brekke et al. (2007), however, we also explore a scenario where pseudo-generics are present. By so doing, we uncover additional results. In particular: (i) as mentioned, RP yields higher consumer surplus than FPR, but the difference between the two is shown to be larger when a pseudo-generic is present in the market; and (ii) RP is shown to be particularly advantageous, from a welfare viewpoint (public payer perspective), when a pseudo-generic is present. This result is inevitably linked to Rodrigues et al. (2011), because in the presence of a pseudo-generic, firms' profits and hence total pharmaceutical expenditure are higher. As firms, under a FPR mechanism, 'mark-up' their prices and profits relative to a RP mechanism, this 'mark-up' is larger, in absolute terms, when a pseudo-generic is present. By eliminating this 'mark-up', reference pricing brings about a larger increase in consumer surplus and welfare.

This paper has the following structure: section 2 describes the base model; sections 3 and 4 analyse the two reimbursement mechanisms in a setting where a pseudo-generic is absent or present respectively; section 5 presents the paper's main results and section 6 concludes.

\section{Model}

The model is based on Rodrigues et al. (2011). We assume two firms, an incumbent (I) and an entrant (E), which compete by simultaneously setting prices. The incumbent sells a branded pharmaceutical product (denoted by $b$ ) and may also sell a generic, non-branded, variety of that product - a pseudo-generic denoted by $p g$. The entrant only sells the generic variety (denoted by g). $B, P G$, and $G$ refer to quantities (or demand functions) of these varieties.

Branded and generic varieties are assumed to be vertically differentiated in the eyes of consumers: consumers have a higher reservation price for the branded variety $(\beta)$ than for the generic $(\gamma)$, i.e. $\beta>\gamma>0$. Horizontal differentiation is also assumed, whereby uniformely distributed consumers must decide on their preferred product along an Hotelling interval $[0,1]$, where the entrant 
is located at 0 and the incumbent at 1 , and where the disutility of consuming a generic is assumed to be linear in the distance to the preferred variety, with slope $t>0$. Consumers will purchase a unit if their surplus $(C S)$ is positive and none otherwise. If the surplus is positive for more than one product variety, the consumer will choose the variety which yields the highest surplus:

$$
C S_{i}=\left\{\begin{array}{cc}
\beta-\hat{p}_{b} & \text { if she buys } b \\
\gamma-\hat{p}_{k}-t \times\left|f_{j}-c_{i}\right|, k=g, p g & \text { if she buys } g \text { or } p g \\
0 & \text { otherwise }
\end{array}\right.
$$

where $\left(\hat{p}_{b}, \hat{p}_{p g}, \hat{p}_{g}\right)$ is the price vector relevant to consumers (which we refer to as effective prices) and $c_{i}$ denotes consumer $i$ 's type, i.e. $c_{i}$ measures the distance between consumer $i$ 's location and the left endpoint of the unit interval, and $f_{j}$ denotes firm $j$ 's location.

We analyse two possible reimbursement schemes: a fixed percentage reimbursement (FPR) mechanism, where consumers must pay the same percentage (copayment rate), $\theta \in[0,1]$, of their desired product varieties' headline price; and a reference pricing (RP) mechanism, where the consumer's copayment is based on the price of the "reference" pharmaceutical, typically the generic. In order to highlight the differences between these two reimbursement mechanisms, under the FPR mechanism the effective prices are given by:

$$
\hat{p}_{k}^{F P R}=p_{k}^{F P R}-(1-\theta) p_{k}^{F P R}=\theta p_{k}^{F P R}, k=b, p g, g
$$

We define $p_{k}^{F P R}(k=b, p g, g)$ to be the headline prices set by firms when the FPR mechanism is in place. By contrast, under a reference pricing mechanism, the effective prices are given by (we assume that the reference pharmaceutical is the generic and that the copayment rate, $\theta$, is similar across mechanims):

$$
\begin{aligned}
& \hat{p}_{k}^{R P}=p_{k}^{R P}-(1-\theta) p_{g}^{R P}, k=b, p g \\
& \hat{p}_{g}^{R P}=p_{g}^{R P}-(1-\theta) p_{g}^{R P}=\theta p_{g}^{R P}
\end{aligned}
$$

where $p_{k}^{R P}(k=b, p g, g)$ are the headline prices set by firms when the RP mechanism is in place.

We will also refer to the no reimbursement (NR) scenario - the setting analyzed by Rodrigues et al. (2011) -, whereby patients must support the headline prices in full; this is equivalent to setting $\theta=1$ in equation (2) or (3). Finally, production costs are assumed to be zero and the total number of consumers is set equal to 1, i.e. product quantities can be interpreted as market shares.

\section{The market with no pseudo-generics}

In this setting, we assume that the incumbent (I) does not sell a pseudo-generic, i.e. it only sells the branded $(b)$ variety, whilst the entrant $(\mathrm{E})$ only sells the generic variety $(g)$. In this case, the 
consumers' effective prices given by equations (2) and (3) do not include the pseudo-generic variety $(p g)$.

\subsection{Fixed Percentage Reimbursement}

The "marginal consumer", $c_{b . g}^{F P R}$, who is indifferent between buying the branded $(b)$ or generic $(g)$ pharmaceuticals is found by solving $\gamma-\theta p_{g}^{F P R}-t \times c_{b . g}^{F P R}=\beta-\theta p_{b}^{F P R}$, which yields:

$$
c_{b . g}^{F P R}=\frac{\theta\left(p_{b}^{F P R}-p_{g}^{F P R}\right)-(\beta-\gamma)}{t}
$$

Let $\mathbf{p}^{F P R}=\left(p_{b}^{F P R}, p_{g}^{F P R}\right)$ represent the price vector. As the total number of consumers is assumed to be equal to 1 , the demand functions are given by:

$$
\begin{aligned}
B\left(\mathbf{p}^{F P R}\right) & =1-c_{b . g}^{F P R}=1-\frac{\theta\left(p_{b}^{F P R}-p_{g}^{F P R}\right)-(\beta-\gamma)}{t} \\
G\left(\mathbf{p}^{F P R}\right) & =c_{b . g}^{F P R}=\frac{\theta\left(p_{b}^{F P R}-p_{g}^{F P R}\right)-(\beta-\gamma)}{t}
\end{aligned}
$$

The profit functions are given by $\Pi_{B}\left(\mathbf{p}^{F P R}\right)=p_{b}^{F P R} \times B\left(\mathbf{p}^{F P R}\right)$ and $\Pi_{G}\left(\mathbf{p}^{F P R}\right)=p_{g}^{F P R} \times$ $G\left(\mathbf{p}^{F P R}\right)$. Maximizing these functions with respect to $p_{b}^{F P R}$ and $p_{g}^{F P R}$ respectively we obtain the best-response functions:

$$
\begin{aligned}
p_{b}^{F P R} & =\frac{1}{2 \theta} t+\frac{1}{2 \theta}(\beta-\gamma)+\frac{1}{2} p_{g}^{F P R} \\
p_{g}^{F P R} & =\frac{1}{2} p_{b}^{F P R}-\frac{1}{2 \theta}(\beta-\gamma)
\end{aligned}
$$

In a Nash equilibrium, we obtain:

$$
\begin{aligned}
& p_{b}^{F P R}=\frac{2}{3 \theta} t+\frac{1}{3 \theta}(\beta-\gamma) \\
& p_{g}^{F P R}=\frac{1}{3 \theta} t-\frac{1}{3 \theta}(\beta-\gamma)
\end{aligned}
$$

Therefore, equilibrium quantities are given by:

$$
\begin{aligned}
B^{F P R} & =\frac{2}{3}+\frac{1}{3 t}(\beta-\gamma) \\
G^{F P R} & =\frac{1}{3}-\frac{1}{3 t}(\beta-\gamma)
\end{aligned}
$$

and equilibrium profits are: 


$$
\begin{aligned}
& \Pi_{B}^{F P R}=\frac{1}{\theta}\left(\frac{4}{9} t+\frac{4}{9}(\beta-\gamma)+\frac{(\beta-\gamma)^{2}}{9 t}\right) \\
& \Pi_{G}^{F P R}=\frac{1}{\theta}\left(\frac{1}{9} t-\frac{2}{9}(\beta-\gamma)+\frac{(\beta-\gamma)^{2}}{9 t}\right)
\end{aligned}
$$

When no reimbursement (NR) mechanism exists, $\theta=1$ and we obtain the results of Rodrigues et al. (2011). Note that $p_{k}^{F P R}=\frac{1}{\theta} p_{k}^{N R}$, with $k=b, g$. Under a fixed percentage reimbursement scheme, firms mark-up their headline prices in an inversely proportional manner relative to the copayment rate: the higher is the copayment rate, the lower is that mark-up under a FPR mechanism. This implies that the effective price paid by consumers is equal in both cases and therefore equilibrium quantities are also the same, i.e. $\hat{p}_{k}^{F P R}=\hat{p}_{k}^{N R}(k=b, g), B^{F P R}=B^{N R}$ and $G^{F P R}=G^{N R}$. Profit levels become $\Pi_{j}^{F P R}=\frac{1}{\theta} \Pi_{j}^{N R}, j=B, G$, i.e. firms also increase their profits in an inversely proportional manner relative to the copayment rate. Headline prices, $p_{b}$ and $p_{g}$, are lowest when the patient's copayment rate is maximal, i.e. when $\theta=1$. This result is similar to that of Brekke et al. (2007).

Consumer surplus (CS) is given by the sum of the surplus of buying the branded and generic pharmaceuticals (in equilibrium, $c_{b . g}^{F P R}=\frac{t+\gamma-\beta}{3 t}$ ):

$$
\begin{aligned}
C S^{F P R} & =\int_{0}^{\frac{t+\gamma-\beta}{3 t}}\left(\gamma-\theta p_{g}^{F P R}-t \times c\right) d c+\int_{\frac{t+\gamma-\beta}{3 t}}^{1}\left(\beta-\theta p_{b}^{F P R}\right) d c \\
& =\frac{(\beta-\gamma)^{2}-11 t^{2}+10 \beta t+8 \gamma t}{18 t}
\end{aligned}
$$

Note that CS does not depend on $\theta$ : as outlined above, the existence of a copayment rate has no impact on the effective prices consumers must face, $\hat{p}_{b}$ and $\hat{p}_{g}$. Hence, the existence of a FPR mechanism (through parameter $\theta$ ) does not affect consumer surplus, i.e. $C S^{F P R}=C S^{N R}$.

Total profits, which are equivalent to total pharmaceutical expenditure, are given by:

$$
\begin{aligned}
\Pi^{F P R} & =\Pi_{B}^{F P R}+\Pi_{G}^{F P R} \\
& =\frac{1}{\theta}\left(\frac{5}{9} t+\frac{2}{9}(\beta-\gamma)+\frac{2}{9} \frac{(\beta-\gamma)^{2}}{t}\right)
\end{aligned}
$$

Government (or other third-party payers) expenditure with pharmaceuticals is a proportion $(1-\theta)$ of total pharmaceutical expenditure:

$$
\begin{aligned}
G^{F P R} & =(1-\theta) \Pi^{F P R} \\
& =\frac{1-\theta}{\theta}\left(\frac{5}{9} t+\frac{2}{9}(\beta-\gamma)+\frac{2}{9} \frac{(\beta-\gamma)^{2}}{t}\right)
\end{aligned}
$$


Parameter restrictions Two restrictions must hold for these results to be valid. Firstly, $0<c_{b . g}^{F P R}<1$, i.e. both the branded and the generic varities are sold in equilibrium. $c_{b . g}^{F P R}<1$ is always verified, whilst for $c_{b . g}^{F P R}>0$ to be verified, the following condition must hold:

$$
\beta<t+\gamma
$$

Secondly, $\beta \geq p_{b}^{F P R}$, i.e. consumer surplus must be positive (in equilibrium) for both product varieties. In order for this to be verified, the following must hold:

$$
\beta \geq \frac{2 t-\gamma}{3 \theta-1}
$$

These restrictions are similar to those of Rodrigues et al. (2011) in a setting where no reimbursement mechanism exists (i.e. $\theta=1) .^{3}$

\subsection{Reference Pricing}

When a reference pricing mechanism is in place, the marginal consumer, indifferent between purchasing a generic or a branded pharmaceutical, is found by setting $\gamma-\hat{p}_{g}^{R P}-t \times c_{b . g}^{R P}=\beta-\hat{p}_{b}^{R P}$, which yields:

$$
c_{b . g}^{R P}=\frac{\left(p_{b}^{R P}-p_{g}^{R P}\right)-(\beta-\gamma)}{t}
$$

Notice that the marginal consumer in this case has the same location as the marginal consumer when no reimbursement mechanism exists (which can be found by setting $\theta=1$ in equation (4)), i.e. $c_{b . g}^{R P}=c_{b . g}^{N R}$. Therefore, demand and profit functions, as well as equilibrium quantities and prices are equal in these two scenarios: $p_{k}^{R P}=p_{k}^{N R}$, with $k=b, g$. Notably, this result does not depend on $\theta$ : regardless of the copayment rate, as long as the patient's copayment is calculated on the basis of the reference pharmaceutical - in this case the generic -, both firms will have incentives to lower their prices to the levels which they would choose if a reimbursement mechanism did not exist. To that extent, in the absence of price regulation of pharmaceuticals, reference pricing is more successful in bringing about price competition than a FPR mechanism.

Consumers surplus under a RP mechanism is given by: ${ }^{4}$

$$
\begin{aligned}
C S^{R P} & =\int_{0}^{\frac{t+\gamma-\beta}{3 t}}\left(\gamma-\theta p_{g}^{R P}-t \times c\right) d c+\int_{\frac{t+\gamma-\beta}{3 t}}^{1}\left(\beta-p_{b}^{R P}+(1-\theta) p_{g}^{R P}\right) d c= \\
& =\frac{(\beta-\gamma)^{2}-(5+6 \theta) t^{2}+(6 \theta+4) \beta t+(14-6 \theta) \gamma t}{18 t}
\end{aligned}
$$

Total profits are given by:

\footnotetext{
${ }^{3}$ For a graphical representation of these restrictions, see Rodrigues et al. (2011).

${ }^{4}$ In equilibrium, the marginal consumer is located at $c_{b . g}^{R P}=\frac{t+\alpha-\beta}{3 t}$
} 


$$
\begin{aligned}
\Pi^{R P} & =\Pi_{B}^{R P}+\Pi_{G}^{R P} \\
& =\frac{5}{9} t+\frac{2}{9}(\beta-\gamma)+\frac{2}{9} \frac{(\beta-\gamma)^{2}}{t}
\end{aligned}
$$

Government expenditure is a proportion $(1-\theta)$ of the reference price for all pharmaceuticals:

$$
\begin{aligned}
G^{R P} & =(1-\theta)\left(B^{R P}+G^{R P}\right) p_{g}^{R P} \\
& =(1-\theta) p_{g}^{R P} \\
& =(1-\theta)\left(\frac{1}{3} t-\frac{1}{3}(\beta-\gamma)\right)
\end{aligned}
$$

Parameter restrictions As outlined earlier, two conditions must be verified: $0<c_{b . g}^{R P}<1$ and $\beta \geq p_{b}^{R P}$. In the first case, the same restriction outlined earlier must also hold: $\beta<t+\gamma$. As for the second case, the following must hold: $\beta \geq t-\gamma / 2$ (the RP mechanism is equivalent to the NR mechanism, i.e. $\theta=1$ in the previous second restriction given by equation (14)).

\section{The market with a pseudo-generic}

When the incumbent, in addition to the branded pharmaceutical, also sells the pseudo-generic, let $f_{I} \in(0,1)$ represent its location.

\subsection{Fixed Percentage Reimbursement}

Solving $\gamma-\hat{p}_{g}^{F P R}-t c_{g . p g}^{F P R}=\gamma-\hat{p}_{p g}^{F P R}-t\left(f_{I}-c_{g . p g}^{F P R}\right)$ we find the location $\left(c_{g . p g}^{F P R}\right)$ of the consumer who is indifferent between the pseudo-generic and the generic:

$$
c_{g \cdot p g}^{F P R}=\frac{f_{I}}{2}+\frac{\theta\left(p_{p g}^{F P R}-p_{g}^{F P R}\right)}{2 t}
$$

Similarly, solving $\gamma-\hat{p}_{p g}^{F P R}-t\left(c_{b . p g}^{r, F P R}-f_{I}\right)=\beta-\hat{p}_{b}^{F P R}$ we find the location $\left(c_{b . p g}^{r, F P R}\right)$ of the consumer who is indifferent between the branded and pseudo-generic products $(r$ indicates that this consumer is located to the right of $f_{I}$ ):

$$
c_{b . p g}^{r, F P R}=f_{I}-\frac{\beta-\gamma}{t}+\frac{\theta\left(p_{b}^{F P R}-p_{p g}^{F P R}\right)}{t}
$$

Let $\underline{\mathbf{p}}^{F P R}=\left(p_{b}^{F P R}, p_{p g}^{F P R}, p_{g}^{F P R}\right)$ be the headline price vector under a FPR mechanism. Demand functions are thus given by: 


$$
\begin{aligned}
B\left(\underline{\mathbf{p}}^{F P R}\right) & =1-c_{b . p g}^{r, F P R}=1-f_{I}+\frac{\beta-\gamma}{t}-\frac{\theta\left(p_{b}^{F P R}-p_{p g}^{F P R}\right)}{t} \\
P G\left(\underline{\mathbf{p}}^{F P R}\right) & =c_{b . p g}^{r, F P R}-c_{g . p g}^{F P R}=\frac{f_{I}}{2}-\frac{\beta-\gamma}{t}+\frac{\theta\left(p_{b}^{F P R}-p_{p g}^{F P R}\right)}{t}-\frac{\theta\left(p_{p g}^{F P R}-p_{g}^{F P R}\right)}{2 t} \\
G\left(\underline{\mathbf{p}}^{F P R}\right) & =c_{g . p g}^{F P R}=\frac{f_{I}}{2}+\frac{\theta\left(p_{p g}^{F P R}-p_{g}^{F P R}\right)}{2 t}
\end{aligned}
$$

In this scenario, the incumbent produces both the branded and the pseudo-generic products. Hence, its profit function is given by $\left[\Pi_{B}\left(\underline{\mathbf{p}}^{F P R}\right)+\Pi_{P G}\left(\underline{\mathbf{p}}^{F P R}\right)\right]=p_{b}^{F P R} \times B\left(\underline{\mathbf{p}}^{F P R}\right)+p_{p g}^{F P R} \times$ $P G\left(\underline{\mathbf{p}}^{F P R}\right)$, whilst the generic producer's profit function is given by $\Pi_{G}\left(\underline{\mathbf{p}}^{F P R}\right)=p_{g}^{F P R} \times$ $G\left(\underline{\mathbf{p}}^{F P R}\right)$. Maximizing the former with respect to $p_{b}^{F P R}$ and $p_{p g}^{F P R}$ and the latter with respect to $p_{g}^{F P R}$, we find the best-response functions which lead to the following Nash equilibrium prices (the underscore is used to differentiate the equibrium prices, quantities and profits in the case with and without a pseudo-generic in the market):

$$
\begin{aligned}
\underline{p}_{b}^{F P R} & =\frac{11}{6 \theta} t-\frac{5}{6 \theta} t f_{I}+\frac{1}{2 \theta}(\beta-\gamma) \\
\underline{p}_{p g}^{F P R} & =\frac{4}{3 \theta} t-\frac{1}{3 \theta} t f_{I} \\
\underline{p}_{g}^{F P R} & =\frac{2}{3 \theta} t+\frac{1}{3 \theta} t f_{I}
\end{aligned}
$$

At these prices, we obtain the equilibrium quantities:

$$
\begin{aligned}
\underline{B}^{F P R} & =\frac{1}{2}\left(1-f_{I}\right)+\frac{\beta-\gamma}{2 t} \\
\underline{P G}^{F P R} & =\frac{1}{6}\left(1+2 f_{I}\right)-\frac{\beta-\gamma}{2 t} \\
\underline{G}^{F P R} & =\frac{1}{3}+\frac{1}{6} f_{I}
\end{aligned}
$$

And the equilibrium profits are:

$$
\begin{aligned}
\underline{\Pi}_{B}^{F P R}+\underline{\Pi}_{P G}^{F P R} & =\frac{1}{36 \theta}\left(t\left(41-f_{I}\left(34-11 f_{I}\right)\right)+18\left(1-f_{I}\right)(\beta-\gamma)+\frac{9(\beta-\gamma)^{2}}{t}\right) \\
\underline{\Pi}_{G}^{F P R} & =\frac{1}{18 \theta} t\left(2+f_{I}\right)^{2}
\end{aligned}
$$

As mentioned earlier, when no reimbursement (NR) mechanism exists, $\theta=1$. Similarly to the results of Section 3.1, $\underline{p}_{k}^{F P R}=\frac{1}{\theta} \underline{p}_{k}^{N R}$, with $k=b, p g, g$ : headline prices are marked-up in an inversely proportional manner relative to the copayment rate, which implies that the effective price paid by consumers and the quantities they purchase are equal in both cases. Profit levels are $\underline{\Pi}_{k}^{F P R}=\frac{1}{\theta} \underline{\Pi}_{k}^{N R}, k=B, P G, G$. 
Consumer surplus is given by: ${ }^{5}$

$$
\begin{aligned}
\underline{C S}^{F P R}= & \int_{0}^{\frac{f_{I}}{6}+\frac{1}{3}}\left(\gamma-\theta \underline{p}_{g}^{F P R}-t \times c\right) d c+\int_{\frac{f_{I}+\frac{1}{3}}{6}}^{f_{I}}\left(\gamma-\theta \underline{p}_{p g}^{F P R}-t \times\left(f_{I}-c\right)\right) d c+ \\
& +\int_{f_{I}}^{\frac{\left(1+f_{I}\right) t-(\beta-\gamma)}{2 t}}\left(\gamma-\theta \underline{p}_{p g}^{F P R}-t \times\left(c-f_{I}\right)\right) d c+\int_{\frac{\left(1+f_{I}\right) t-(\beta-\gamma)}{2 t}}^{1}\left(\beta-\theta \underline{p}_{b}^{F P R}\right) d c \\
= & \frac{9(\beta-\gamma)^{2}+(18 \beta+54 \gamma) t-18(\beta-\gamma) t f_{I}-\left(115-86 f_{I}+61 f_{I}^{2}\right) t^{2}}{72 t}
\end{aligned}
$$

Notice that $\underline{C S}^{F P R}$ does not depend on $\theta$ because effective prices paid by consumers do not depend on the existence of a copayment rate. Hence, $\underline{C S}^{F P R}=\underline{C S}^{N R}$.

Total profits are given by:

$$
\begin{aligned}
\underline{\Pi}^{F P R} & =\underline{\Pi}_{B}^{F P R}+\underline{\Pi}_{P G}^{F P R}+\underline{\Pi}_{G}^{F P R} \\
& =\frac{1}{36 \theta}\left(t\left(49-f_{I}\left(26-13 f_{I}\right)\right)+18\left(1-f_{I}\right)(\beta-\gamma)+\frac{9(\beta-\gamma)^{2}}{t}\right)
\end{aligned}
$$

Government expenditure is given by:

$$
\begin{aligned}
\underline{G}^{F P R} & =(1-\theta) \underline{\Pi}^{F P R} \\
& =\frac{(1-\theta)}{36 \theta}\left(t\left(49-f_{I}\left(26-13 f_{I}\right)\right)+18\left(1-f_{I}\right)(\beta-\gamma)+\frac{9(\beta-\gamma)^{2}}{t}\right)
\end{aligned}
$$

Parameter restrictions Five conditions must hold for these results to be valid. Firstly, $0<c_{b . g}^{F P R}<1$, which is equivalent to requiring that:

$$
\gamma+\left(\frac{1}{3}-\frac{7}{3} f_{I}\right) t<\beta<\gamma+\frac{7}{3}\left(1-f_{I}\right) t
$$

Secondly, $c_{b . p g}^{r, F P R}>c_{b . g}^{F P R}$ and $\gamma-\underline{\hat{p}}_{p g}^{F P R}-t f_{I}<\gamma-\underline{\hat{p}}_{g}^{F P R}$, i.e. the pseudo-generic is sold in equilibrium. The latter is satisfied provided $\underline{\hat{p}}_{p g}^{F P R}>\underline{\hat{p}}_{g}^{F P R}$, which holds in equilibrium. The former is satisfied provided:

$$
f_{I}>2 / 5
$$

If this condition holds, the first inequality in equation (28) is always satisfied for any $\beta>\gamma$.

Thirdly, $f_{I}<c_{b . p g}^{r, F P R}<1$, so that the branded product is sold in equilibrium. $c_{b . p g}^{r, F P R}<1$ is always satisfied, but in order for $f_{I}<c_{b . p g}^{r, F P R}$ the following must hold:

\footnotetext{
${ }^{5}$ In equilibrium, $c_{g \cdot p g}^{F P R}=\frac{f_{I}}{6}+\frac{1}{3}$ and $c_{b . p g}^{F P R}=\frac{\left(1+f_{I}\right) t-(\beta-\gamma)}{2 t}$.
} 


$$
\beta<\gamma+\left(1-f_{I}\right) t
$$

Fourthly, $c_{b . g}^{F P R}>c_{b . p g}^{l, F P R}$ so that the pseudo-generic is sold in equilibrium. This is equivalent to requiring that:

$$
\beta<\gamma+\left(\frac{5}{3}-\frac{8}{3} f_{I}\right) t
$$

This condition is more restrictive than the second inequality in equation (28) and the inequality in equation (30). In addition, this condition sets an upper boundary for $f_{I}$, because by assumption $\beta>\gamma$. Hence, for this assumption to hold, $f_{I}<5 / 8$. Together with equation (29), this implies that:

$$
2 / 5<f_{I}<5 / 8
$$

Finally, all product varieties must provide positive surplus, which is equivalent to requiring that $\beta \geq \underline{\hat{p}}_{b}^{F P R}$. This is verified provided the following condition holds:

$$
\beta \geq \frac{11 t-5 t f_{I}-3 \gamma}{6 \theta-3}
$$

Therefore, equations (31), (32) and (33) must hold for our results to be verified. ${ }^{6}$

\subsection{Reference Pricing}

It is straightforward to show that the location of consumer who is indifferent between the branded and pseudo-generic products and between the pseudo-generic and the generic products is equivalent to the NR scenario. ${ }^{7}$ Hence, equilibrium quantities, prices and profits are equal to those in that scenario (namely $\underline{p}_{k}^{R P}=\underline{p}_{k}^{N R}$, with $k=b, p g, g$, and the latter can be found by setting $\theta=1$ in equation (22)).

Consumer surplus is given by: ${ }^{8}$

\footnotetext{
${ }^{6}$ With the exception of equation (33), all other restrictions are similar to those of Rodrigues et al. (2011), where their graphical representation can also be found.

${ }^{7}$ Solving $\gamma-\hat{p}_{g}^{R P}-t c_{g . p g}^{R P}=\gamma-\hat{p}_{p g}^{R P}-t\left(f_{I}-c_{g . p g}^{R P}\right)$ we obtain the latter $\left(c_{g . p g}^{R P}=\frac{f_{I}}{2}+\frac{p_{p g}^{R P}-p_{g}^{R P}}{2 t}\right)$, whilst solving $\gamma-\hat{p}_{p g}^{R P}-t\left(c_{b . p g}^{r, R P}-f_{I}\right)=\beta-\hat{p}_{b}^{R P}$ we find the former $\left(c_{b . p g}^{r . R P}=f_{I}-\frac{\beta-\gamma}{t}+\frac{p_{b}^{R P}-p_{p g}^{R P}}{t}\right)$.

${ }^{8}$ In equilibrium, $c_{g . p g}^{R P}=\frac{f_{I}}{6}+\frac{1}{3}$ and $c_{b . p g}^{R P}=\frac{\left(1+f_{I}\right) t-(\beta-\gamma)}{2 t}$.
} 


$$
\begin{aligned}
\underline{C S}^{R P}= & \int_{0}^{\frac{f_{I}}{6}+\frac{1}{3}}\left(\gamma-\theta \underline{p}_{g}^{R P}-t \times c\right) d c+\int_{\frac{f_{I}}{6}+\frac{1}{3}}^{f_{I}}\left(\gamma-\theta \underline{p}_{p g}^{R P}+(1-\theta) \underline{p}_{g}^{R P}-t \times\left(f_{I}-c\right)\right) d c+ \\
& +\int_{f_{I}}^{\frac{\left(1+f_{I}\right) t-(\beta-\gamma)}{2 t}}\left(\gamma-\theta \underline{p}_{p g}^{R P}+(1-\theta) \underline{p}_{g}^{R P}-t \times\left(c-f_{I}\right)\right) d c+ \\
& +\int_{\frac{\left(1+f_{I}\right) t-(\beta-\gamma)}{2 t}}^{1}\left(\beta-\theta \underline{p}_{b}^{R P}+(1-\theta) \underline{p}_{g}^{R P}\right) d c \\
= & \frac{9(\beta-\gamma)^{2}+(18 \beta+54 \gamma) t-18(\beta-\gamma) t f_{I}-\left(67+48 \theta-110 f_{I}+24 \theta f_{I}+61 f_{I}^{2}\right) t^{2}}{72 t}
\end{aligned}
$$

Total profits are given by:

$$
\begin{aligned}
\underline{\Pi}^{R P} & =\underline{\Pi}_{B}^{R P}+\underline{\Pi}_{P G}^{R P}+\underline{\Pi}_{G}^{R P} \\
& =\frac{1}{36}\left(t\left(49-f_{I}\left(26-13 f_{I}\right)\right)+18\left(1-f_{I}\right)(\beta-\gamma)+\frac{9(\beta-\gamma)^{2}}{t}\right)
\end{aligned}
$$

Recall that total profits under a RP mechanism are equivalent to total profits under a NR mechanism, i.e. $\underline{\Pi}^{R P}=\underline{\Pi}^{N R}$.

Government expenditure is given by:

$$
\begin{aligned}
\underline{G}^{R P} & =(1-\theta)\left(\underline{B}^{R P}+\underline{P G}^{R P}+\underline{G}^{R P}\right) \underline{p}_{g}^{R P} \\
& =(1-\theta) \underline{p}_{g}^{R P} \\
& =(1-\theta)\left(\frac{2}{3} t+\frac{1}{3} t f_{I}\right)
\end{aligned}
$$

Parameter restrictions As in the FPR mechanism case, equations (31), (32) and (33) must hold for our results to be verified. In the latter case of equation (33), the condition is less restrictive because of the equivalence between the RP and NR mechanisms, which implies that $\theta=1$ in equation (33).

\section{$5 \quad$ Market impact of the pseudo-generic}

Rodrigues et al. (2011) show, in their Proposition 1, that the presence of a pseudo-generic raises the prices of all pharmaceuticals in a setting with no reimbursement mechanism. We extend this result by claiming that:

Proposition 1 For parameter values satisfying equations (31), (32) and (33), the presence of a pseudo-generic raises the headline price of the branded and generic varieties under both reimbursement regimes (FPR and $R P$ ). 
Proof. Under a FPR mechanism with $\theta>0, \underline{p}_{b}^{F P R}>p_{b}^{F P R}$ is equivalent to requiring that $\beta>\gamma-7 t+5 t f_{I}$; in order for $\underline{p}_{g}^{F P R}>p_{g}^{F P R}, \beta>\gamma-t+t f_{I}$. Both hold for any $\beta>\gamma, t>0$ and $f_{I} \in(0,1)$.

Under a RP mechanism, headline prices are equivalent to a no reimbursement scenario, as assumed in Rodrigues et al. (2011). As demonstrated in their proof of Proposition $1, \underline{p}_{b}^{N R}=\underline{p}_{b}^{R P}>$ $p_{b}^{N R}=p_{b}^{R P}$ and $\underline{p}_{g}^{N R}=\underline{p}_{g}^{R P}>p_{g}^{N R}=p_{g}^{R P}$ for any $\beta>\gamma, t>0$ and $f_{I} \in(0,1)$.

From a consumer's perspective, although the headline prices are not fully borne because of the reimbursement mechanisms, we can still state that:

Proposition 2 Under both reimbursement mechanisms (FPR and RP), and assuming equations (31), (32) and (33) hold, consumer surplus is lower when a pseudo-generic is present in the market.

Proof. Under a FPR mechanism, consumer surplus with a pseudo-generic is given by equation (10), whilst equation (25) contains the expression for consumer surplus with no pseudo-generic. In order for $\underline{S}^{F P R}-C S^{F P R}<0$, the following must be verified:

$$
\underline{C S}^{F P R}-C S^{F P R}=\frac{(\beta-\gamma)\left[5(\beta-\gamma)-22 t-18 t f_{I}\right]-\left(71-86 f_{I}+61 f_{I}^{2}\right) t^{2}}{72 t}<0
$$

The denominator is always positive; hence, a sufficient condition for the expression to be negative is for both terms in the numerator to be negative. Starting with the second term, $\left(71-86 f_{I}+61 f_{I}^{2}\right) t^{2}$, it is always negative for any $t>0$ and $f_{I} \in(0,1)$; as for the first term, $(\beta-\gamma)>0$ (by assumption) and in order for $5(\beta-\gamma)-22 t-18 t f_{I}<0$ the following must hold:

$$
\beta<\gamma+\frac{22}{5} t+\frac{18}{5} t f_{I}
$$

This is less restrictive than equation (31) and hence always satisfied when equation (31) is active.

Under a RP mechanism, consumer surplus with and without a pseudo-generic is given by equations (34) and (16) respectively. $\underline{C S}^{R P}-C S^{R P}$ is given by:

$$
\underline{C S}^{R P}-C S^{R P}=\frac{(\beta-\gamma)\left[5(\beta-\gamma)+(2-24 \theta) t-18 t f_{I}\right]-\left(47+24 \theta-110 f_{I}+24 \theta f_{I}+61 f_{I}^{2}\right) t^{2}}{72 t}
$$

The denominator is always positive; the second term in the numerator is always negative for any $\theta>1$ and $2 / 5<f_{I}<5 / 8$, as equation (32) is active; in order for the first term to be negative, the following must hold:

$$
\beta<\gamma-\left(\frac{2}{5}-\frac{24}{5} \theta\right) t+\frac{18}{5} t f_{I}
$$


This is less restrictive than equation (31) provided $f_{I}>31 / 94$, which is satisfied when the restriction embodied in equation (32) is active.

Reference pricing, from the consumers viewpoint, is clearly better than the FPR mechanism, but it is particularly advantageous when a pseudo-generic is sold in the market:

Proposition 3 Assuming equations (31), (32) and (33) hold, reference pricing yields a higher consumer surplus than a fixed percentage reimbursement mechanism, but the difference between the two is larger when a pseudo-generic is present in the market.

Proof. Reference pricing leads to a higher consumer surplus than the FPR mechanism, as we can see from the following expressions::

$$
\begin{aligned}
C S^{R P}-C S^{F P R} & =\frac{1-\theta}{3}[t-(\beta-\gamma)]>0 \\
\underline{C S}^{R P}-\underline{C S}^{F P R} & =\frac{t(1-\theta)\left(f_{I}+2\right)}{3}>0
\end{aligned}
$$

The first expression is clearly positive because equation (13) holds, i.e. $(\beta-\gamma)<t$; the second expression is always positive for any $t>0, \theta<1$ and $f_{I} \in(0,1)$. The rationale is straightforward: headline prices are equal under a RP or a NR mechanism, but in the latter the consumer supports the full price of pharmaceuticals whilst under RP he only supports part of it, thus lowering effective prices.

Comparing the increases in consumer surplus arising from the introduction of reference pricing (relative to a FPR mechanism) in equation (41), we obtain the following result:

$$
\left(\underline{C S}^{R P}-\underline{C S}^{F P R}\right)-\left(C S^{R P}-C S^{F P R}\right)=\frac{1-\theta}{3}\left[t+(\beta-\gamma)+t f_{I}\right]
$$

This expression is positive for any $\theta<1, t>0, \beta>\gamma$ and $f_{I} \in(0,1)$.

This result suggests, from a consumer's viewpoint, that there is particular merit in introducing reference pricing when a pseudo-generic is present, as this leads to a more significant increase in consumer surplus (relative to a FPR mechanism). Alternatively, within a FPR mechanism, this result indicates a more pressing need for price regulation when a pseudo-generic is present.

\section{Welfare analysis}

Inevitably, the relative merits of each reimbursement mechanism should not be assessed solely by looking at their effects on consumer surplus. Social welfare (SW) - the sum of consumer and producer surplus minus government expenditure with pharmaceuticals - is a more encompassing welfare measure. 
Proposition 4 Assuming equations (31), (32) and (33) hold, social welfare in a setting with no pseudo-generic is equal regardless of the reimbursement mechanism; the same is true in a setting with a pseudo-generic, i.e. $S W^{N R}=S W^{F P R}=S W^{R P}$ and $\underline{S W^{N R}}=\underline{S W^{F P R}}=\underline{S W^{R P}}$ respectively.

Proof. Without a pseudo-generic, social welfare in the case of a FPR mechanism is given by $S W^{F P R}=C S^{F P R}+\Pi^{F P R}-G^{F P R}$. As we can see from equation (10), $C S^{F P R}=C S^{N R}$, because consumer surplus does not depend on $\theta$. Additionally, $\Pi^{F P R}=\frac{1}{\theta} \Pi^{N R}$ and $G^{F P R}=\frac{1-\theta}{\theta} \Pi^{N R}$. Hence:

$$
\begin{aligned}
S W^{F P R} & =C S^{F P R}+\Pi^{F P R}-G^{F P R} \\
& =C S^{N R}+\frac{1}{\theta} \Pi^{N R}-\frac{1-\theta}{\theta} \Pi^{N R} \\
& =C S^{N R}+\Pi^{N R}=S W^{N R}
\end{aligned}
$$

Under reference pricing, equation (41) tells us that $C S^{R P}=C S^{F P R}+\frac{1-\theta}{3}[t-(\beta-\gamma)]=$ $C S^{N R}+\frac{1-\theta}{3}[t-(\beta-\gamma)]$. As we have seen in Section 3.2, total profits under reference pricing are equal to a no reimbursement scenario, i.e. $\Pi^{R P}=\Pi^{N R}$. Finally, government expenditure is given by equation (18). Therefore:

$$
\begin{aligned}
S W^{R P} & =C S^{R P}+\Pi^{R P}-G^{R P} \\
& =C S^{N R}+\frac{1-\theta}{3}[t-(\beta-\gamma)]+\Pi^{N R}-\frac{(1-\theta)}{3}[t-(\beta-\gamma)] \\
& =C S^{N R}+\Pi^{N R}=S W^{N R}
\end{aligned}
$$

When a pseudo-generic is present, $\underline{S W}^{F P R}=\underline{C S}^{F P R}+\underline{\Pi}^{F P R}-\underline{G}^{F P R}$. From equation (25), we know that $\underline{C S}^{F P R}=\underline{C S}^{N R}$. Equation (26) tells us that $\underline{\Pi}^{F P R}=\frac{1}{\theta} \underline{\Pi}^{N R}$ whilst government expenditure, from equation (27), is simply $\underline{G}^{F P R}=\frac{1-\theta}{\theta} \underline{\Pi}^{N R}$. Hence:

$$
\begin{aligned}
\underline{S W}^{F P R} & =\underline{C S}^{F P R}+\underline{\Pi}^{F P R}-\underline{G}^{F P R} \\
& =\underline{C S}^{N R}+\frac{1}{\theta} \underline{\Pi}^{N R}-\frac{1-\theta}{\theta} \underline{\Pi}^{N R} \\
& =\underline{C S}^{N R}+\underline{\Pi}^{N R}=\underline{S W}
\end{aligned}
$$

Under reference pricing, equation (41) tells us that $\underline{C S}^{R P}=\underline{C S}^{F P R}+\frac{t(1-\theta)\left(f_{I}+2\right)}{3}=\underline{C S}^{N R}+$ $\frac{t(1-\theta)\left(f_{I}+2\right)}{3}$ whilst equation (35) tells us that $\underline{\Pi}^{R P}=\underline{\Pi}^{N R}$. Finally, government expenditure is given by equation (36), which yields: 


$$
\begin{aligned}
\underline{S W}^{R P} & =\underline{C S}^{R P}+\underline{\Pi}^{R P}-\underline{G}^{R P} \\
& =\underline{C S}^{N R}+\frac{t(1-\theta)\left(f_{I}+2\right)}{3}+\underline{\Pi}^{N R}-\frac{t(1-\theta)\left(f_{I}+2\right)}{3} \\
& =\underline{C S}^{N R}+\underline{\Pi}^{N R}=\underline{S W^{N R}}
\end{aligned}
$$

This result is similar to Brekke et al. (2007) (Proposition 6). With or without a pseudo-generic, it is particularly interesting to note that, under a FPR mechanism, pharmaceutical firms effectively receive a public subsidy but consumers do not benefit from lower (effective) prices: the mark-up of headline prices is fully borne by the government and hence this transfer (effectively a public subsidy) is neutral from a social welfare perspective. This result clearly indicates the need for pharmaceutical price regulation prior to inclusion in a FPR mechanism.

By contrast, under reference pricing, it is consumers who receive a public subsidy, which allows them to increase their consumer surplus because they no longer bear the full headline price of pharmaceuticals. From a social welfare perspective, this transfer is also neutral, because firms' profits are equal to a NR scenario.

Brekke et al. (2007) suggest a different welfare measure which may be more relevant to countries which do not have a significant pharmaceutical industry. In those cases, it is unlikely that profits would enter the welfare function. Thus, welfare $(W)$ can be more narrowly defined in a public payer perspective, only taking into account consumer surplus net of government expenditure. Following this approach, we can state that:

Proposition 5 Assuming equations (31), (32) and (33) hold, $W^{N R}=W^{R P}>W^{F P R}$ and $\underline{W}^{N R}=$ $\underline{W}^{R P}>\underline{W}^{F P R}$, i.e. a FPR mechanism is worse than RP or NR from a public payer's welfare perspective. In addition, reference pricing is particularly advantageous relative to FPR when a pseudo-generic is present in the market, i.e. $\left(\underline{W}^{R P}-\underline{W}^{F P R}\right)>\left(W^{R P}-W^{F P R}\right)$.

Proof. In a setting with no pseudo-generic, $W^{F P R}=C S^{F P R}-G^{F P R}=C S^{N R}-\frac{1-\theta}{\theta} \Pi^{N R}$ whilst $W^{R P}=C S^{R P}-G^{R P}=C S^{N R}$ (see proof of Proposition 4). Hence, $W^{N R}=W^{R P}>W^{F P R}$.

When a pseudo-generic is present, $\underline{W}^{F P R}=\underline{C S}^{F P R}-\underline{G}^{F P R}=\underline{C S}^{N R}-\frac{1-\theta}{\theta} \underline{\Pi}^{N R}$, whereas $\underline{W}^{R P}=\underline{C S}^{R P}-\underline{G}^{R P}=\underline{C S}^{N R}$ (see proof of Proposition 4) and hence $\underline{W}^{N R}=\underline{W}^{R P}>\underline{W}^{F P R}$.

We know that $\left(\underline{W}^{R P}-\underline{W}^{F P R}\right)=\frac{1-\theta}{\theta} \underline{\Pi}^{R P}$ and $\left(W^{R P}-W^{F P R}\right)=\frac{1-\theta}{\theta} \Pi^{R P}$. Therefore, in order for $\left(\underline{W}^{R P}-\underline{W}^{F P R}\right)>\left(W^{R P}-W^{F P R}\right)$, it is sufficient to demonstrate that $\underline{\Pi}^{R P}>\Pi^{R P}$, provided $0<\theta<1$ :

$$
\underline{\Pi}^{R P}-\Pi^{R P}=\frac{1}{36 t}\left[29 t^{2}+10 t(\beta-\gamma)-26 t^{2} f_{I}+13 t^{2} f_{I}^{2}-18 t f_{I}(\beta-\gamma)+(\beta-\gamma)^{2}\right]
$$


The latter term is always positive. Hence, the expression is positive provided $29 t^{2}+10 t(\beta-\gamma)-$ $26 t^{2} f_{I}+13 t^{2} f_{I}^{2}-18 t f_{I}(\beta-\gamma)>0$. For $f_{I}<5 / 9$ this is equivalent to requiring that:

$$
\beta>\gamma+\frac{t\left(-29+26 f_{I}-13 f_{I}^{2}\right)}{10-18 f_{I}}
$$

The denominator is positive for $f_{I}<5 / 9$, but the numerator is always negative and hence any $\beta>\gamma$ satisfies this inequality. When $f_{I}>5 / 9$,requiring that $29 t^{2}+10 t(\beta-\gamma)-26 t^{2} f_{I}+13 t^{2} f_{I}^{2}-$ $18 t f_{I}(\beta-\gamma)>0$ is equivalent to requiring that:

$$
\beta<\gamma+\frac{t\left(-29+26 f_{I}-13 f_{I}^{2}\right)}{10-18 f_{I}}
$$

This is less restrictive than equation (31) and hence always satisfied if this restriction is active.

Intuitively, reference pricing brings about a more significant increase in welfare from a public payer's perspective (or, equivalently, a more significant reduction in government expenditure), compared to a FPR mechanism, when a pseudo-generic is present. This is because under a FPR mechanism, as we have seen, prices and profits are 'marked-up' relative to a RP mechanism. If, as we show, total profits (or total pharmaceutical expenditure) are higher when a pseudo-generic is present, then this 'mark-up' will necessarily be higher in absolute terms. Hence, the introduction of reference pricing, by eliminating this 'mark-up', will bring about a more significant increase in welfare in that setting.

\section{Conclusion}

This paper extends the analysis of Rodrigues et al. (2011) by looking at two widely adopted reimbursement mechanisms: a fixed percentage reimbursement mechanism and a reference pricing mechanism. In particular, in a setting encompassing both vertical product differentiation (between branded and non-branded pharmaceuticals) and horizontal product differentiation (between generics) a number of interesting results are uncovered. First, under either reimbursement mechanism, the results of Rodrigues et al. (2011) hold: the presence of a pseudo-generic raises the prices of all pharmaceuticals and can be used as a tool to soften competition between the branded and generic pharmaceutical producers. In addition, reference pricing is shown to bring about lower prices and thus higher consumer surplus than FPR mechanisms, but this effect is more significant when a pseudo-generic is present. Second, from a welfare perspective, our results are equivalent to those of Brekke et al. (2007): if firms' profits are excluded from the analysis - a public payer's welfare perspective in countries where the pharmaceutical industry is absent - reference pricing is also superior to FPR. Lastly, we further show that this difference is larger when a pseudo-generic is present, which implies that, in this case, adopting reference pricing would be particularly advantageous. 
Alternatively, if a pseudo-generic is present, this suggests a more pressing need for price regulation within a FPR mechanism.

This analysis can be extended to a third possible, and to the best of our knowledge yet underresearched, reimbursement mechanism: asymmetric fixed percentage reimbursement, through which the reimbursement rates are different for different types of pharmaceuticals, with generics typically attracting higher reimbursement (lower copayment) rates. This type of mechanism has been used, for instance, in Portugal in order to help increase generic penetration at an early development stage. Whilst this type of mechanism introduces more complexity in the analysis, it may also have the merit to uncover more intricate details of firms' pricing incentives.

In addition, when a pseudo-generic is present, we have assumed that transportation costs (or product substitutability) are equal for all generics (including the pseudo-generic). This may not be a plausible assumption: generally, the identity of the producer is visible in the product and the pseudo-generic may thus be considered, by consumers, to be 'better' (closer to the branded product) than other generics. These are likely to be the next steps in our research.

\section{References}

[1] Brekke, K., Königbauer, I. and Straume, O. (2007), "Reference pricing of pharmaceuticals", Journal of Health Economics, 46, 613-642.

[2] Brekke, K., Grasdal, A. and Holmå, T. (2009), "Regulation and pricing of pharmaceuticals: Reference pricing or price cap regulation?", European Economic Review, 53, 170-185.

[3] López-Casasnovas and Puig-Junoy, J. (2000), "Review of the literature on reference pricing", Health Policy, 54, 87-123.

[4] Merino-Castelló, A. (2003), "Impact of the Reference Pricing Systemon the Pharmaceutical Market: A Theoretical Approach", Universitat Pompeu Fabra, Working Paper 524a.

[5] Miraldo, M. (2009), "Reference pricing and firms' pricing strategies", Journal of Health Economics, $28,176-197$.

[6] Rodrigues, V., Gonçalves, R. and Vasconcelos, H. (2011), "Anticompetitive impact of pseudogenerics", mimeo. 